\title{
The association between lung function and arterial stiffness in young childhood
}

\author{
To the Editor:
}

Several studies have shown that reduced lung function is a risk factor for cardiovascular morbidity and mortality [1]. Precursors for cardiovascular diseases, like hypertension and arterial stiffness, are also associated with lower lung function, independent of sex or anthropometrics [2-6]. Currently, there is no explanation for these relationships. Systemic inflammation or traditional cardiovascular risk factors, such as smoking and serum cholesterol, could not fully explain this relationship [4,5]. Recent findings support the hypothesis that a common pathway exists between a decline in lung function and, subsequently, the development of arterial stiffness or hypertension [3, 6]. Suggested explanations were shared factors, like infections during childhood or postnatal growth, which could influence both lung function and arterial stiffness. However, little is known about the association between arterial stiffness and lung function, or about the influence of anthropometrics and environmental factors in early life. In this study, we aimed to assess whether an association between lung function and common carotid artery stiffness is already present in young healthy children.

Participants of this study were children of WHISTLER (Wheezing Illnesses Study Leidsche Rijn), an ongoing birth cohort [7]. During visits at the ages of 5 and 8 years, information on the child and parents was gathered by a questionnaire, and weight and height were measured. Spirometry was performed conform the latest American Thoracic Society/European Respiratory Society statements [8]. Ultrasonography of the a. carotis communis was performed according to previously described methods [9]. Both a lower distensibility and a higher elastic modulus indicate a stiffer artery. This study was approved by the Medical Ethics Committee of the University Medical Centre Utrecht (Utrecht, the Netherlands). Written informed parental consent was obtained.

Linear regression analyses were performed to study the association between lung function as the independent variable and vascular parameters as the dependent variable. In multivariable linear regression analyses, we assessed the influence of anthropometrics and environmental factors in separate models. Missing data on determinants were imputed using multiple imputations. Pooled results of 10 imputed datasets are expressed as linear regression coefficients with $95 \%$ confidence intervals.

Within this study, 553 5-year-old children and 230 8-year-old children had a successful lung function and vascular measurement. Table 1 shows the linear regression coefficients for the association of lung function and vascular parameters in the different models. In the univariable model, there was a relation between lower forced expiratory volume in $1 \mathrm{~s}(\mathrm{FEV} 1)$ and increased arterial stiffness (higher distensibility and lower elastic modulus). After adjustment for age, sex, weight and height, there was no statistically significant association anymore (model 2). Adjustment for early environmental factors, and adjustment for smoking and experienced infections or allergies, in models 3 and 4, respectively, did not affect the association. The results at the age of 8 years were similar to those at 5 years. The models with forced vital capacity as the dependent variable showed similar results in the 5 -year-old children. The linear regression coefficients had a similar trend in the 8-year-olds, but did not reach statistical significance (data not shown).

In total, 184 children had a successful spirometry at the age of 5 and 8 years, and a vascular measurement at the age of 8 years. A larger increase in FEV1 $\left(\Delta \mathrm{FEV}_{1}\right)$ from age 5 to 8 years was associated with increased arterial stiffness (linear regression coefficients $-25.42 \mathrm{MPa}^{-1} \cdot \mathrm{L}^{-1}\left(95 \% \mathrm{CI}-42.42--8.42 \mathrm{MPa}^{-1} \cdot \mathrm{L}^{-1}\right.$ ) and $63.96 \mathrm{kPa} \cdot \mathrm{L}^{-1},\left(95 \% \mathrm{CI} 19.51-108.4 \mathrm{kPa} \cdot \mathrm{L}^{-1}\right)$ for distensibility and elastic modulus, respectively). After adjustment for sex, weight and height at 8 years, the associations were no longer statistically significant $\left(-14.65 \mathrm{MPa}^{-1} \cdot \mathrm{L}^{-1}\left(95 \% \mathrm{CI}-32.04-2.73 \mathrm{MPa}^{-1} \cdot \mathrm{L}^{-1}\right)\right.$ and $33.30 \mathrm{kPa} \cdot \mathrm{L}^{-1}\left(95 \% \mathrm{CI}-11.34-77.93 \mathrm{kPa} \cdot \mathrm{L}^{-1}\right)$ for distensibility and elastic modulus, respectively). Adjustment for environmental factors alone did not affect the association.

Our study provides evidence that, in childhood, higher lung function is associated with higher arterial stiffness. This association was largely explained by anthropometry, while environmental factors alone did not affect the association. 
TABLE 1 Association between lung function and arterial stiffness

Age 5 years

Age 8 years

$\begin{array}{cc}\text { Distensibility } \mathrm{MPa}^{-1} & \\ \text { Model 1 } & -17.89(-30.27--5.50) \\ \text { Model 2 } & -7.67(-23.34-8.00) \\ \text { Model 3 } & -17.48(-29.94--5.01) \\ \text { Model 4 } & -17.76(-30.21--5.30) \\ \text { Elastic modulus kPa } & \\ \text { Model 1 } & 35.08(12.60-57.55) \\ \text { Model 2 } & 19.49(-8.77-47.75) \\ \text { Model 3 } & 35.32(12.69-57.94) \\ \text { Model 4 } & \mathbf{3 4 . 4 3}(\mathbf{1 1 . 8 2 - 5 7 . 0 5})\end{array}$

$-17.75(-28.26--7.25)$

$-4.04(-17.56-9.49)$

$-16.71(-27.26--6.17)$

$-18.35(-28.94--7.76)$

47.17 (18.84-75.50)

$7.89(-28.46-44.24)$

$46.44(17.87-75.02)$

$47.29(18.85-75.74)$

Data are presented as linear regression coefficients representing difference in vascular outcomes per litre increase in forced expiratory volume in $1 \mathrm{~s}$ based on the imputed datasets. Model 1: no adjustments; model 2: adjusted for age, sex, height and weight; model 3: adjusted for smoke exposure of the mother during pregnancy and 3 months postnatal growth; model 4: adjusted for current smoking of one of the parents, infections and allergy diagnosed by a general practitioner. Bold indicates statistical significance.

To interpret the results of this study, some issues need to be considered. Some of our data are self reported and possibly imprecise. For instance, parental smoking was self reported, although our data do seem to reflect real smoking habits [10]. However, certainly not all measured (developmental) factors were self reported. The visits to general practitioner were extracted from electronic medical records and information about postnatal growth was prospectively gathered [11]. Although we cannot exclude the possibility of residual confounding, we do believe to have maximally addressed this issue, given the measurements that we have available.

We feel that our study has several strengths. To our best knowledge, our vascular measurements at the age of 5 years are the youngest reported in a large group of healthy children. Given the complexity of measurements, our sample size is substantial. Moreover, our study adds to insights on the association between individual change in lung function and arterial wall characteristics.

Our findings are consistent with other studies. Recently, we performed a similar study to study the relationship between blood pressure (including pulse pressure, an indirect marker of arterial stiffness) and lung function from neonatal to elderly age. This study showed that, in young individuals, higher lung function is associated with higher blood pressure. This association reversed with increasing age [12]. This finding was consistent with another cross-sectional study performed in adults [4]. However, findings opposite to our current results also have been presented. A study among 8-year-old children showed that lower lung volumes are associated with increased arterial stiffness, measured by the augmentation index [13]. The different findings from that study and ours are most likely due to differences in participants and methodology. The participants of that study were at high familial risk of developing asthma. Selection on asthma predisposition could have induced predominance of both lower lung volumes and stiffer arteries.

Currently, it is unknown whether properties of the vascular system in the young have the same pathophysiological implications as in adults. Probably, the association between lower lung function and higher arterial stiffness described in adulthood develops later in life. The biological pathway linking respiratory function and arterial stiffness is still unknown, but as shown by our study, in childhood, this is largely explained by anthropometry and not environmental factors. Based on previous literature [14], it can be speculated that the reversal of the association might be due to prolonged exposure to pulmonary and cardiovascular risk factors. A second explanation might be tissue stiffening (ageing) of both the pulmonary and vascular systems over time. Third, the changes in pulmonary and vascular system over time might be directly causally related. Longer follow-up is needed to give a better understanding of this reversal.

It is known that early-life adverse exposures could lead to permanent metabolic or structural changes, which in later life result in cardiovascular disease [15]. From a preventive point of view, it is important to get a better understanding of the early life origins of this association. It is probably too soon for practical purposes but there is a need for studies on early origins of associations that are becoming quite clear in later life. 
This is the first study exploring the association between lung function and arterial stiffness in a large group of healthy children, measured at two different ages. In conclusion, our study provides evidence that the association between impaired lung function and increased arterial stiffness, as described in adulthood, is not present in childhood, but probably emerges later in life. The univariable association between higher lung function and increased arterial stiffness is lost after adjusting for age, sex, weight and height.

@ERSpublications

In childhood, higher lung function is associated with higher arterial stiffness http://ow.ly/v3HaL

Jacobien B. Eising ${ }^{1}$, Cornelis K. van der Ent ${ }^{1}$, Annemieke M.V. Evelein ${ }^{2}$ and Cuno S.P.M. Uiterwaal ${ }^{2}$

${ }^{1}$ Dept of Paediatric Pulmonology, Wilhelmina Children's Hospital, University Medical Centre, Utrecht, The Netherlands.

${ }^{2}$ Julius Centre for Health Sciences and Primary Care, University Medical Centre Utrecht, Utrecht, The Netherlands.

Correspondence: Jacobien B. Eising, Paediatric Pulmonology Dept, Wilhelmina Children's Hospital, Room KH.01.419.0, Lundlaan 6, P.O. Box 85090, 3508 AB Utrecht, The Netherlands. E-mail: j.eising@umcutrecht.nl

Received: Dec 272013 | Accepted after revision: March 122014 | First published online: May 152014

Support statement: The Wheezing Illnesses Study Leidsche Rijn received grant 2100.0095 from The Netherlands Organisation for Health Research and Development (ZonMw). An unrestricted grant was received from Glaxo Smith Kline. Additionally, this work was financially supported by the UMC Utrecht Vascular Prevention Project.

Conflict of interest: None declared.

Acknowledgements: The authors gratefully acknowledge all parents and children who participated, Caroline Geerts and Liesbeth van der Feltz-Minkema (University Medical Centre (UMC), Utrecht, the Netherlands) for their major contribution to the data collection, Myriam Olling-de Kok (UMC, Utrecht) for secretarial support, Jildou Zwerver for data management and the Vascular Imaging Centre (UMC, Utrecht) for assistance in the vascular measurements.

\section{References}

1 Engstrom G, Lind P, Hedblad B, et al. Lung function and cardiovascular risk: relationship with inflammationsensitive plasma proteins. Circulation 2002; 106: 2555-2560.

2 Barr RG, Ahmed FS, Carr JJ, et al. Subclinical atherosclerosis, airflow obstruction and emphysema: the MESA Lung Study. Eur Respir J 2012; 39: 846-854.

3 Bolton CE, Cockcroft JR, Sabit R, et al. Lung function in mid-life compared with later life is a stronger predictor of arterial stiffness in men: the Caerphilly Prospective Study. Int J Epidemiol 2009; 38: 867-876.

4 Jankowich MD, Taveira T, Wu W-C. Decreased lung function is associated with increased arterial stiffness as measured by peripheral pulse pressure: data from NHANES III. Am J Hypertens 2010; 23: 614-619.

5 Zureik M, Benetos A, Neukirch C, et al. Reduced pulmonary function is associated with central arterial stiffness in men. Am J Respir Crit Care Med 2001; 164: 2181-2185.

6 Jacobs DR, Yatsuya H, Hearst MO, et al. Rate of decline of forced vital capacity predicts future arterial hypertension: the Coronary Artery Risk Development in Young Adults Study. Hypertension 2012; 59: 219-225.

7 Katier N, Uiterwaal CSPM, de Jong BM, et al. The Wheezing Illnesses Study Leidsche Rijn (WHISTLER): rationale and design. Eur J Epidemiol 2004; 19: 895-903.

8 Beydon N, Davis SD, Lombardi E, et al. An official American Thoracic Society/European Respiratory Society statement: pulmonary function testing in preschool children. Am J Respir Crit Care Med 2007; 175: 1304-1345.

9 Geerts CC, Evelein AMV, Bots ML, et al. Body fat distribution and early arterial changes in healthy 5-year-old children. Ann Med 2012; 44: 350-359.

10 Geerts CC, Bots ML, Van Der Ent CK, et al. Parental smoking and vascular damage in their 5-year-old children. Pediatrics 2013; 129: 45-54.

11 Evelein AMV, Visseren FLJ, van der Ent CK, et al. Excess early postnatal weight gain leads to increased abdominal fat in young children. Int J Pediatr 2012; 2012: 141656.

12 Eising JB, van der Ent CK, van der Gugten AC, et al. Life-course of cardio-respiratory associations. Eur J Prev Cardiol 2013 [In press DOI: 10.1177/2047487313510410].

13 Ayer JG, Belousova EG, Harmer JA, et al. Lung function is associated with arterial stiffness in children. PLoS One 2011; 6: e26303.

14 Sin DD, Wu L, Man SFP. The relationship between reduced lung function and cardiovascular mortality: a population-based study and a systematic review of the literature. Chest 2005; 127: 1952-1959.

15 Barker DJ. Fetal origins of coronary heart disease. BMJ 1995; 311: 171-174. 